\title{
Evolution of colon cancer and pregnancy: a report of two cases
}

Keywords: colon cancer, bevacizumab, capecitabine, necrobiosis, immunotolerance, inmunosupression

\section{Introduction}

Colon cancer and pregnancy is a very few frequent disease. We found scarce specific articles about this association. ${ }^{1}$ The diagnosis is difficult because except for bleeding signs, the symptoms could be mimetized by the signs and symptoms of the pregnancy in itself. There are few reports about these cases. Makoshi et al. ${ }^{2}$ reported a case treated with FOLFOX-6 to a pregnant woman between 22 and 30 gestational weeks. The patient tolerated well the treatment and was delivered of a term newborn without any harm. Ossendorp et al. ${ }^{3}$ assisted a pregnant woman who complained for abdominal pain and finally they diagnosed a colon cancer in advanced stage performing a cesarean section and left side hemi colectomy. We present two cases assisted in our Department and analyzed their evolution.

\section{Case one}

A 30-year-old woman who in September 2014 was diagnosed with advanced colon adenocarcinoma undergoing a 27-week's pregnancy. The patient presented tumor apparently of adnexal origin so a laparotomy was done performing anexectomy with capsular injury of the cyst during the procedure. The pathological anatomy reveals mucinous adenocarcinoma of intestinal origin. In later statification studies, sigma mass and hepatic metastasis were observed with identical pathological results.

Before to start a treatment, a decision about to the pregnancy had to be done. We met with the general and oncological surgeons, oncologists and obstetricians. A decisión to continue the evolution of pregnancy was encouraged by us, because nobody could said that the patient were to did worse being pregnant because the colon cancer and the chemo was not going to promote malformations at this gestational age. May be the fetus could develop a growth restriction, but we have all the necessary methods and techniques to assist him/her and to deliver the fetus if we consider indicated, taken in account the extremely preemy and very low birth weight and the sequelae percentage expectable if we deliver this fetus at 27 week's of gestational age. So, she received steroids for fetal lung maturation and started chemo under very close obstetrical control of pregnancy evolution.

She started chemotherapy, performing four cycles of FOLFOX (oxaliplatin-folinic acid-5 fluorouracil) regimen with partial response. In December 2014, the cesarean section was performed at 37weeks of gestational age in agreement with neonatologists; it was delivered a newborn male, 3000grams, Apgar 9/10. During C Section, we observed the colon disease in sigmoid, and there were observed some pieces of fibrotic tissue in the uterus that seems us metastasis and were removed and sent to pathological evaluation. The general surgeons found by palpation MTS in liver.

In January of the year 2015 a progression with multiple peritoneal

\author{
Volume 3 Issue I - 2017
}

\author{
Illia R,' Bas C, ${ }^{2}$ Rotholtz N, ${ }^{3}$ Cabrera M, ${ }^{4}$ \\ Lobenstein $G,{ }^{5}$ Uranga Imaz $M,{ }^{5}$ Manrique \\ $\mathrm{G},{ }^{5}$ Fiameni $\mathrm{F}^{5}$ \\ 'Chief of Obstetrics Service, Professor of Obstetrics at Buenos \\ Aires University, Argentina \\ ${ }^{2}$ Chief of Oncology Service, Buenos Aires University, Argentina \\ ${ }^{3}$ Chief of Surgery Service, Buenos Aires University, Argentina \\ ${ }^{4}$ Chief of Residents at Obstetrics \& Gynecology Service, Buenos \\ Aires University, Argentina \\ ${ }^{5}$ Obstetrical staff phycisians, Buenos Aires University, Argentina
}

Correspondence: Ricardo Illia, Chief of Obstetrics Service, Professor of Obstetrics at Buenos Aires University, FACOG, Argentina,Email rhillia@gmail.com

Received: October 28, 2017 | Published: November 09, 2017

implants was confirmed. Unexpectedly, the patient developed a fecaloid peritonitis for which it was restarted to chemotherapy and performed four cycles of FOLFOX, with 3cycles of Bevacizumab.

Left hepatectomy due to disease progression was performed in June 2015. Re-initiation of chemotherapy with 3 cycles of FOLFOXIRI (oxaliplatin-folinic acid-5 fluorouracil-irinotecan)+Bevacizumab régimen resulted in a partial response.

In October of the year 2015 was performed resection of hepatic and adnexal metastases and restarted chemotherapy with FOLFOXIRI scheme. Adnexal progression and uronephrosis are observed. Chemotherapy resumes for 4cycles: FOLFOXIRI+Bevacizumab regimen.

In June of the year 2016, hepatic relapse is performed with resection of liver metastases.

Subsequently continued with Capecitabine + Bevacizumab.

She could breastfeed the baby and she is still alive and her baby is going to turn on two years old. Obviously that her prognosis is possibly ominous in face of the future, but with this medical strategie, she survived, the newborn was delivered close to term, is healthy and at least, is still under the care of his mother. On the other way round, we have had a very preterm newborn with all the problems attributable to extreme prematurity without any improvement in the mother's health (Figure 1).

\section{Case two}

A 37-years-old patient started with non-specific abdominal pain in the year 2013 in an 8-week's pregnancy context. She presented abdominal ultrasound evidencing: multiple isoecogenic nodules in the left lobe and in the right hepatic lobe with central necrobiosis. $9 \mathrm{~mm}$ thickening of the hepatic angle of the colon and reduction of light of 
$8 \mathrm{~mm}$ with ascending colon dilation. Video colonoscopy: progresses to rectum-sigma from where cannot progress longer. Hepatic lesions were punctured. Pathological anatomy reveals: malignant lesion compatible with primary colon tumor.

In June of the year 2013 was performed a right hemicolectomy at 12 weeks of pregnancy. Pathological anatomy reports: Colon Adenocarcinoma. It was classified as stage IV due to liver metastases. Once again we opted by medical treatment with close obstetrical control of pregnancy.
FOLFOX scheme (oxaliplatin-folinic acid-5 fluorouracil) was proposed and started.

Unexpectedly, the patient presented spontaneous abortion at 20 weeks of gestational age without any apparent or evidenciable cause, and nobody knows the potential relation between the main disease, the oncological treatment and the final outcome of the pregnancy.

Continue with FOLFOX scheme later and the patient passed away few month later (Figure 2).
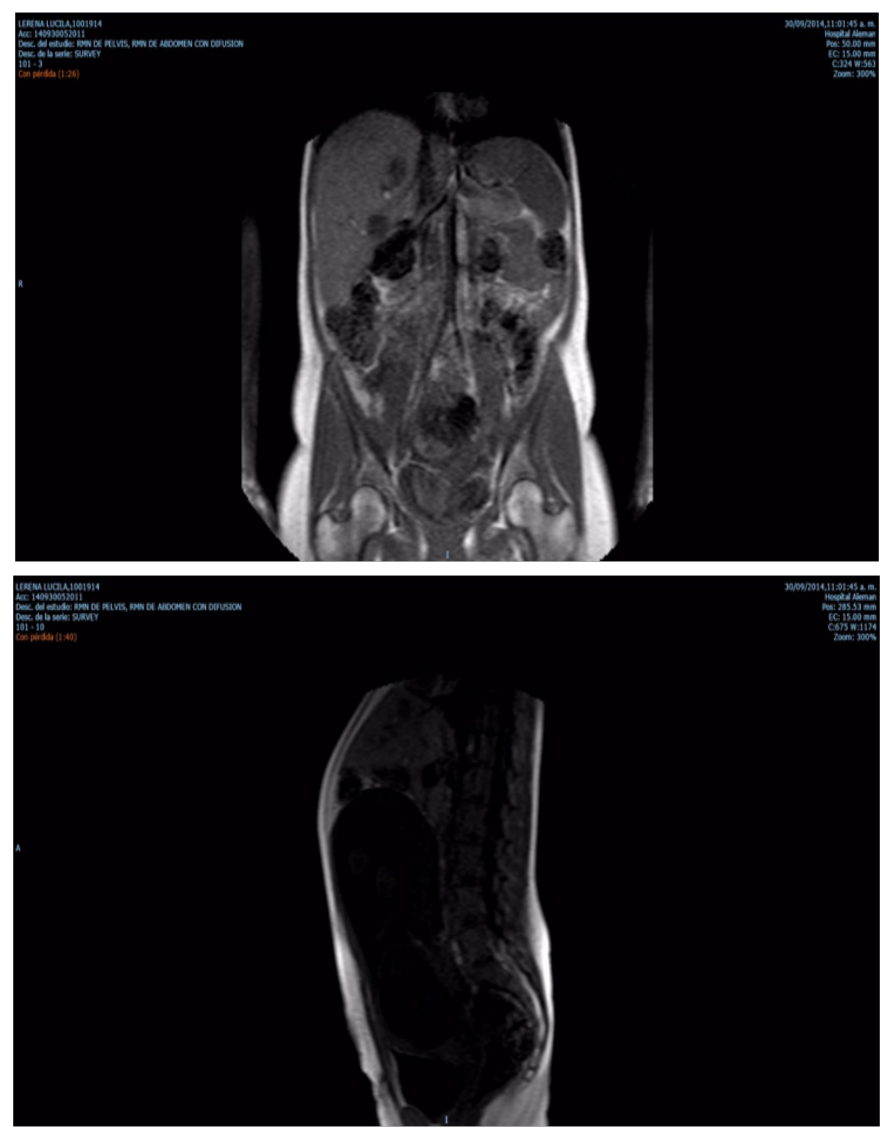

Figure I Liver, Focal images hypointenses in TI and hyperintenses en en T2 related to MTS. Uterus, gestational uterus.

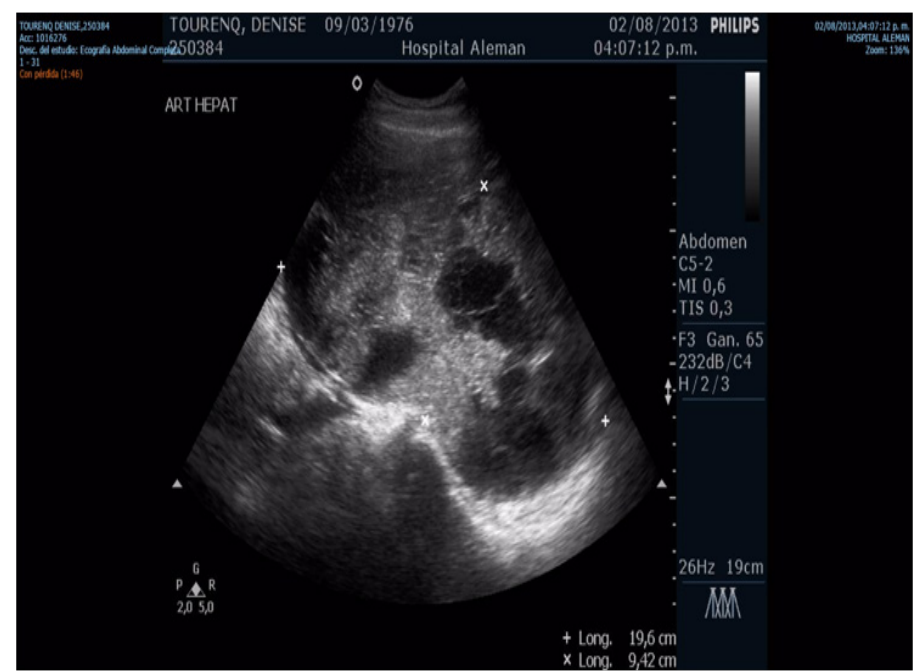

Figure 2 Liver, enlargement because multiple nodular solid images attributables to MTS. 


\section{Comments}

Currently cancer is one of the leading causes of death in women of childbearing age ${ }^{4}$ and there are an increased rate from the 40 years old. This fact makes it possible for an obstetrician to face a pregnant woman with cancer.

With the social changes observed lately at the social level with women privileging their insertion into the labor market or professional development before maternity, there had been an increased age in which they looks for pregnancy. Cancer is a pathology that with the aging of the individual increases their probability of happen. Many malignancies increase their incidence after the age of 35years old, therefore, it is expected that the association between cancer and pregnancy is becoming more frequent. Besides the general situation, our cases may have been carriers of a Lynch Syndrome (wich is an autosomal dominant syndrome that could be suspected in front of a strong family story), because mainly of their youth at the disease appears, but regrettably we cannot check it. ${ }^{5,6}$ Another risk factor to be taken in account in our country, is the high intake of meat. ${ }^{7,8}$ When facing a pregnant woman with cancer the most paramount thing is to have a multidisciplinary team to achieve a right diagnosis, correct staging and the most effective treatment. Such results would have to be comparable to when the cancer is treated outside of pregnancy. ${ }^{9}$ From an obstetrical point of view, both the timely and safe resolution of childbirth and fetal well-being must be ensured in the sense that neither the cancer nor its treatment seriously affects the condition of the fetus and the newborn. Obviously, is not wise to administrate chemotherapy during first trimester, but there are a lot of drugs that can be used after fetal development. ${ }^{10,11}$ The most important complication could be a fetal growth restriction, but, while it would be only a restriction and not a lack of fetal growth and the Doppler evaluations reassured the fetal state, is possible to continue the pregnancy to the most advanced gestational age as possible, as we have done with this two cases of colon cancer and we have done with other cancers, specially breast cancer and pregnancy.

In the face of a malignant neoplastic disease that occurs during pregnancy, two key questions must be considered. Firstly, how pregnancy can affect cancer behavior and secondly, how cancer and its treatment can affect pregnancy (the case two incognito).

It has been suggested that pregnancy can affect the biological behavior of cancer. To this could contribute the hormonal changes and the state of immunotolerance or immuno supression proper to the pregnancy. However, to date there are no studies demonstrating such a hypothesis although is very possible.

Both, the suspicion and the diagnostic confirmation of a malignancy are affected by pregnancy. Many signs and symptoms typical of a neoplastic disease such as precocious satiety, vomiting, alterations in the intestinal transit are also common in pregnancy, mainly at the very beginning. Another element that undermines the early diagnosis of cancer during pregnancy is the doctor's reluctance to request imaging studies, particularly radiological studies.

Another disadvantage that the treating team of this type of patients must face is the establishment of a plan of effective and safe treatment at the same time. Respect to this last comment, we insist in it is not useful for nobody to get a very preterm newborn taking in account all the complications that this baby's develop. Is wise not to forget the high incidences of lung problems, necrotizing enterocolitis because of a blood redistribution, retinal, hearing and neurological and developmental problems, besides they belong to the group that feed the most high frequency of neonatal mortality in Intensive Care Unit.

A comprehensive task in a multidisciplinary team, can help both, physicians and patients to achieve the best outcome possible in spite of to be in front of such serious disease as cancer.

\section{Acknowledgements}

None.

\section{Conflict of interest}

Author declares that there is no conflict of interest.

\section{References}

1. Heise RH, Van Winter JT, Wilson TO, et al. Colonic cancer during pregnancy: case report and review of the literature. Mayo Clin Proc. 1992;67(12):1180-1184

2. Makoshi Z, Perrott C, Al-Khatani K, et al. Chemotherapeutic treatment of colorectal cancer in pregnancy: case report. J Med Case Rep. 2015;9:140

3. Ossendorp RR, Silvis R, van der Bij GJ. Advanced colorectal cancer resulting in acute bowel obstruction during pregnancy; a case report. Eddy DM. Screening for colorectal cancer. Ann Intern Med. 1990;8:18-20.

4. Ahnen DJ, Wade SW, Jones WF, et al. The increasing incidence of young-onset colorectal cancer: a call to action. Mayo Clin Proc. 2014;89(2):216.

5. Dozois EJ, Boardman LA, Suwanthanma W, et al. Young-onset colorectal cancer in patients with no known genetic predisposition: can we increase early recognition and improve outcome? Medicine (Baltimore). 2008;87(5):259

6. Chao A, Thun MJ, Connell CJ, et al. Meat consumption and risk of colorectal cancer. JAMA. 2005;293(2):172.

7. Chan DS, Lau R, Aune D, et al. Red and processed meat and colorectal cancer incidence: meta-analysis of prospective studies. PLoS One. 2011;6:e20456.

8. Giardiello FM, Allen JI, Axilbund JE, et al. Guidelines on genetic evaluation and management of Lynch syndrome: a consensus statement by the US Multi-Society Task Force on Colorectal Cancer. Dis Colon Rectum. 2014;57(8):1025

9. Yoh T, Yamamichi K, Oishi M, et al. A case of effective neoadjuvant chemoradiotherapy with capecitabine for locally advanced sigmoid colon cancer. Gan To Kagaku Ryoho. 2011;38(6):1021.

10. Foxtrot Collaborative Group. Feasibility of preoperative chemotherapy for locally advanced, operable colon cancer: the pilot phase of a randomised controlled trial. Lancet Oncol. 2012;13(11):1152.

11. Makoshi Z, Perrott C, Al-Khatani K, et al. Chemotherapeutic treatment of colorectal cancer in pregnancy: case report 11.Ann Med Surg (Lond). 2016;8:18-20 